\title{
A fresh look at zebrafish from the perspective of cancer research
}

\author{
Shuai Zhao', Jian Huang ${ }^{1 *}$ and Jun $\mathrm{Ye}^{2^{*}}$
}

\begin{abstract}
Zebrafish represent a vertebrate model organism that has been widely, and increasingly, employed over the last decade in the study of developmental processes, wound healing, microbe-host interactions, and drug screening. With the increase in the laboratory use of zebrafish, several advantages, such as a high genetic homology to humans and transparent embryos, which allow clear disease evaluation, have greatly widened its use as a model for studying tumor development in vivo. The use of zebrafish has been applied in several areas of cancer research, mainly in the following domains: (1) establishing cancer models by carcinogenic chemical, genetic technology, and xenotransplantation; (2) evaluating tumor angiogenesis; (3) studying tumor metastasis; and (4) anti-tumor drug screening and drug toxicity evaluation. In this study, we provide a comprehensive overview of the role of zebrafish in order to underline the advantages of using them as a model organism in cancer research. Several related successful events are also reviewed.
\end{abstract}

Keywords: Zebrafish, Cancer model, Angiogenesis, Metastasis, Drug screen

\section{Introduction}

Danio rerio, better known as zebrafish, have emerged as a popular model for studying developmental processes and human disorders. Zebrafish share a high level of genetic and physiologic homology with humans, including brain, digestive tract, musculature, vasculature, and an innate immune system [1-6]. Moreover, approximately $70 \%$ of all human disease genes have functional homologs with the species [7]. Zebrafish are prolific reproducers with the potential to produce over 100 embryos per clutch. Their extrauterine development is rapid; the major organs of the zebrafish are fully developed by 24 hours post fertilization (HPF), and they are ready for use in larvae experiments by 3 days post fertilization (DPF). Zebrafish larvae are transparent during the early stages of life (through to 7 DPF), and this phase can be extended to 9-14 DPF by the addition of melanin synthesis inhibitor [8]. Zebrafish are small in size and require inexpensive food. It is easy, therefore, to maintain thousands of larvae in a laboratory at a reasonable cost.

\footnotetext{
* Correspondence: Drhuangjian@zju.edu.cn; wzmcyejun@zju.edu.cn ${ }^{1}$ Department of Surgical oncology, The Second Affiliated Hospital, Zhejiang University School of Medicine, Hangzhou, Zhejiang Province, China ${ }^{2}$ Department of Gastroenterology, The Second Affiliated Hospital, Zhejiang University School of Medicine, Hangzhou, Zhejiang Province, China
}

Due to the advantages of genetic homology, physiology, and developmental similarity, zebrafish have increasingly become a desirable tool for studying the development and modeling of human disease $[9,10]$. In the transparent embryo and larvae, clear time-lapse non-invasive imaging and protein/cell marker tracking significantly aid the observation of biological and disease processes $[11,12]$. Several types of gastrointestinal disorders, such as inflammatory bowel disease, non-alcoholic fatty liver disease (NAFLD), and alcoholic liver disease, can be modeled in zebrafish [13-17]. Zebrafish have also been used in the analysis of complex brain disorders and muscle disease, including depression [18], autism [19], psychoses [20], and muscular dystrophies [21]. In addition, the ability to regenerate both fins and cardiac tissue make zebrafish particularly suitable for studying the wound healing response to various injuries [22].

Because of these advantages, zebrafish have proved to be superior for use in cancer research over the last decade. There are several long-standing methods for establishing a cancer model in zebrafish, including carcinogenic treatment, transgenic regulation, and the transplantation of mammalian tumor cells [23]. By inducing different gene mutations or activating signaling pathways through the use of chemicals, tumors can be induced in a wide variety 
of organs in zebrafish, such as the liver, pancreas, intestinal canal, skin, muscle, vasculature, and testis [24-28]. Transgenic technology enables the formation of specific types of tumor by the overexpression of particular oncogenes. The xenotransplantation of mammalian tumor cells into zebrafish provides a novel way of studying the interactions between the transplanted tumor cells and the host's vasculature. Zebrafish have also been exploited for the investigation of tumor angiogenesis, which represents a critical step in tumor progression and is a target for anti-tumor therapies. The vascular system in a zebrafish embryo bears a strong resemblance to that in humans, and rapidly forms a single blood circulatory loop at $24 \mathrm{HPF}$. In zebrafish, the vascular endothelial cells can be stained by a fluorescent protein so that the neovascularization in the tumor microenvironment can be observed in the earliest stage. Tumor metastasis has also been modeled in zebrafish. The fluorescent-stained tumor cells are highlighted in the transparent zebrafish embryos and larvaes, so that the process of metastasizing tumor cells can be accurately tracked at the cellular level. The novel casper zebrafish line, a generation of double pigmentation mutant, even has a completely transparent body in adulthood. This superior generation of zebrafish, in conjunction with fluorescent imaging techniques, allows the noninvasive tracing of stained tumor cells in adult fishes [8]. It is worth mentioning that cancer stem cells account for only a small fraction of tumor cells and are too few in number to be feasibly transplanted in a mammalian model in order to assess metastasis. However, only a very small number of cancer stem cells are required in zebrafish for this purpose because of their small size. Additionally, the high fertility and low maintenance costs of zebrafish makes them suitable for the large-scale screen of antineoplastic drug efficacy and toxicity.

This paper focuses attention on the wide application of zebrafish as a superior model in cancer research, particularly with regard to establishing tumor models, and studying angiogenesis, metastasis, and antineoplastic drug screens.

\section{Cancer model establishment in zebrafish}

Neoplasia was rarely found in wild zebrafish. Using a combination of chemical treatment, genetic technology, and tumor cell xenotransplantation, the vast majority of human tumors can be modeled in zebrafish [29]. Carcinogenic chemical treatment is commonly used in inducing tumorigenesis. Several carcinogenic compounds are able to induce canceration in a number of organs, such as dimethylbenzanthracene (DMBA) [30], diethylnitrosamine (DEN) [23], N-nitrosodimethylamine (NDMA) [31], N-ethyl-N-nitrosourea (ENU) [24], and N-methyl-
$\mathrm{N}^{1}$-nitro-N-nitrosoguanidine (MNNG) [32]. The induced tumors cover a wide spectrum of tumors found not only in the digestive system (i.e. liver, pancreas, and intestinal canal) but also in the skin, muscle, vasculature, and testis [24-28]. As reported, exposure of the $v h l^{+/}$ zebrafish to DMBA revealed an increase in the occurrence of hepatic, bile duct, and intestinal tumorigenesis at 2 months following treatment [33]. Exposure to DEN results in different types of hepatocellular carcinomas, hepatoblastomas, hepatoma, cholangiocarcinoma, and pancreatic carcinoma in zebrafish [26]. Exposure of zebrafish to NDMA for 2 months leads to cholangiolar tumors (cholangiocarcinomas and cholangiomas) and hepatocellular tumors (hepatocellular carcinomas and adenomas) [31]. And exposure of zebrafish to ENU and MNNG results in liver and testis tumorigenesis [24, 27].

A number of reverse genetic tools have been developed for the study of gene functions in zebrafish. Morpholinos are usually injected at the 1-4 cell stage of embryos to provide transient knockdown of the target gene expression [34]. Another targeted genome modification technology, called TILLING (Targeting Induced Local Lesions IN Genomes), is highly dependent on large-scale traditional post-transcriptional forward genetic screens expression [35-38]. Moreover, engineered endonucleases, including ZFNs (zinc finger nucleases), the CRISPR-Cas system, and TALENs (transcription activator-like effector nucleases), provide efficient strategies to disrupt sitedirected genes by inducing double strand breaks in the target genes $[39,40]$.

Several types of tumor have been generated by inducing mutants in known tumor suppressor genes. The knockout $p 53$ gene in zebrafish, for example, was found to result in an increase of malignant peripheral nerve sheath tumors (MPNST) [41]. In addition, the APC gene mutant in zebrafish leads to colon adenoma initiation and progression, suggesting an association with the activation of the Wnt signaling pathway [42]. Several other gene mutants were found to be related to different types of tumors in zebrafish. As reported, mutants in the NF1 gene lead to high-grade gliomas and MPNSTs [43], those in BRCA2, mybl2, and espl1 lead to testicular neoplasias $[44,45]$, those in the pen/lgl2, $b m y b$ and $c d s$ genes cause epidermal neoplasia [32, 46, 47], and GSTT1 deletion related to lymphoma progression [48], and $v h l$ mutants lead to an increase in hepatic and intestinal tumors [33]. The immune and hematopoietic system in zebrafish is similar to that in humans, which means that not only solid tumors but also hematologic malignancies can be modeled [6]. The most frequent mutant in the tumor suppressor pten in zebrafish was related to an increasing morbidity of T-cell acute lymphoblastic leukemia (T-ALL) and hemangiosarcoma $[49,50]$. 
Through the transgenic expression of human or mouse oncogenes, several cancer models have been established in zebrafish. T cell acute lymphoblastic leukemia was the first cancer induced by transgenic technology in zebrafish, which was induced by the Myc transgenes [51]. Subsequently, overexpression of the oncogenes $x m r k, M y c$ and $K R A S^{(V 12)}$ in zebrafish was found lead to hepatoma formation in both juvenile and adult transgenic fish [52-55]. Amplification of $M Y C N$ and $f g f 8$ expressions markedly promotes the formation of neuroblastoma [56]. Rhabdomyosarcoma has also been induced in zebrafish by using a specific up-regulate oncogenic $K R A S^{(\mathrm{G} 12 \mathrm{D})}$ expression [57]. Overexpression of $A k t 1$ enhances lipoma formation [58]. In combination with the $p 53$ mutant, overexpression of some oncogenes in zebrafish leads to different tumor phenotypes, such as scr (hepatoma) [59], NRAS (melanoma) [60], BRAF (melanoma) [41], and EWS-FIL1 (Ewing's sarcoma) [61]. Additionally, the co-activation of the hedgehog and AKT pathways promotes tumorigenesis, suggesting that a transgenic approach is a useful tool for studying the interaction of oncogenes and oncogenic pathways in zebrafish [62].

Xenotransplantation represents a novel method to establish tumor models in zebrafish. One of the great strengths of xenotransplantation is that the transplanted tumor cells can be marked by fluorescent staining to enable them to be distinguished from normal cells in order to allow clear observation of the development process of the tumor [63]. The first human xenotransplant assays in zebrafish began in 2005. By injecting $1 \sim 100$ melanoma cells into $3.5 \sim 4.5 \mathrm{HPF}$ embryos, the migration in the developing larvae was clearly observed [64]. Transplantation of different types of tumor cells in zebrafish was carried out subsequent to this innovative work. Microinjecting glioma stem cells into the embryonic yolk sac region in 2 DPF embryos resulted in an observable invasion in the embryos via the vessels [65]. Hepatocellular carcinoma (HCC) was also modeled for the identification of the curative effect of anti-cancer molecules [66]. Several other types of tumor, such as lung cancer [67], pancreatic cancer [68], ovarian carcinomas [69], breast cancer [70], prostate cancer [71], retinoblastoma [72], and leukemia [73], have also been transplanted in zebrafish.

All the methods and types of induced tumor are combined in Table 1 . The induced tumors are mainly located in the digestive and reproductive systems, and then the nervous system and epithelium.

\section{Tumor angiogenesis in zebrafish}

Angiogenesis is considered a key factor in tumor growth and subsequent metastasis. Tumor vessels play an important role in transporting oxygen and nutrients to support the growth of tumor cells. For this reason, the capability of blood vessel formation within the tumor not only determines the malignancy of the cancer but also influences the therapeutic effects and prognosis. Both in research evidence and clinically, angiogenesis inhibitors in combination with chemotherapy improved the outcomes in cancer patients [74]. However, it is difficult to detect the original vascularization in traditional mammalian models because such models only permit the capture of static images, which probably relate to the late stage of the tumor. The lack of observation at the earliest stages of tumor formation means that the mechanism of vascularization is still not fully understood.

Human umbilical vein endothelial cells (HUVEC) are widely used in the investigation of angiogenic mechanisms in vitro. The system of angiogenesis can be evaluated by the cellular responses of HUVEC, such as cell proliferation, cell cycle, tube formation, cell migration, and cell adhesion to matrix proteins [75]. Several other quantitative angiogenesis assays, for instance the matrix implant assay and microcirculatory preparations such as the chicken chorioallantoic membrane and corneal micropocket assay, provide continuous monitoring of the angiogenic response [76]. However, the physiological status of angiogenesis may be quite different when translated to the area of cancer research. Indeed, angiogenesis in the tumor microenvironment relys on a distinct signaling pathway and displays large alterations in morphology and function when compared with normal vasculogenesis. Thus, in vitro research may be not suitable for modeling angiogenesis in tumor organization.

Zebrafish provide an ideal in vivo model for the research of tumor angiogenesis. The physiology and pathology of tumor angiogenesis in zebrafish is similar to that in humans because the tumor microenvironment in zebrafish is strikingly similar [77]. Additionally, the zebrafish vasculature grows rapidly (a single blood circulatory loop in zebrafish is fully developed in 24 HPF) and the transparent body allows for high-resolution in vivo non-invasive imaging [77]. The addition of PTU (a tyrosinae inhibitor that prevents melanin synthesis) to water can lengthen the transparency of the larvae to 9-14 DPF [78].This years, a pigmentation mutant casper line with a completely transparent body has allowed the non-invasive imaging of the vasculature across the whole body [8]. Real-time observation of vessels in larvae can be achieved after microinjection of chemical dyes into the vascular system [79]. Additionally, taking advantage of the $\mathrm{Tg}(\mathrm{flk1}$ : EGFP) zebrafish, a transgenic fish line with a green fluorescent protein tissue-specific expression in the vasculature, individual cell growth and vessel formation can be easily detected under confocal microscopy [80]. In red fluorescent tumor tissues, the green fluorescent protein marked neovascularization is high lighted and enables the observation of angiogenesis in the initial stages. 
Table 1 Summary of the methods used and the types of tumor induced in zebrafish

\begin{tabular}{|c|c|c|c|}
\hline Technology & Treatment & Types of induced tumor & Reference \\
\hline \multirow[t]{5}{*}{ Chemical treatment } & DMBA & hepatoma, cholangiocarcinoma and intestinal cancer & [30] \\
\hline & DEN & hepatoma, cholangiocarcinoma and pancreatic carcinoma & {$[23]$} \\
\hline & NDMA & hepatoma and cholangiocarcinoma & [31] \\
\hline & ENU & hepatoma and testicular cancer & {$[24]$} \\
\hline & MNNG & hepatoma and testicular cancer & [32] \\
\hline \multicolumn{4}{|l|}{ Genetic technology } \\
\hline \multirow[t]{8}{*}{ Knockout: } & P53 & malignant peripheral nerve sheath tumors & {$[41]$} \\
\hline & $A P C$ & colon adenoma & [42] \\
\hline & NF1 & gliomas and malignant peripheral nerve sheath tumors & [43] \\
\hline & BRCA2, MYBL2, esp 11 & testicular cancer & {$[44,45]$} \\
\hline & pen $/ \lg / 2$, bmyb and cds gene & epidermal cancer & {$[32,46,47]$} \\
\hline & GSTT1 & lymphoma & {$[48]$} \\
\hline & $v h l$ & hepatoma and intestinal cancer & {$[33]$} \\
\hline & pten & T-cell acute lymphoblastic leukemia and hemangiosarcoma & {$[49,50]$} \\
\hline \multirow[t]{8}{*}{ Overexpression: } & Myc & T-cell leukemia and hepatoma & {$[51,53]$} \\
\hline & $x m r k$ and $K R A S^{(V 12)}$ & hepatoma & {$[52,54,55]$} \\
\hline & MYCN and fgf8 & neuroblastoma & [56] \\
\hline & $K R A S^{(G 12 D)}$ & rhabdomyosarcoma & {$[57]$} \\
\hline & Akt1 & lipoma & [58] \\
\hline & Scr in p53 mutant background & hepatoma & {$[59]$} \\
\hline & NRAS, BRAF in p53 mutant background & melanoma & {$[60,41]$} \\
\hline & EWS-FIL1 in p53 mutant background & Ewing's sarcoma & {$[61]$} \\
\hline Xenotransplantation & Transplant tumor cells in zebrafish & $\begin{array}{l}\text { Melanoma, glioma, hepatoma, lung cancer, pancreatic cancer, ovarian } \\
\text { carcinomas, breast cancer, prostate cancer, retinoblastoma, leukemia }\end{array}$ & [64-73] \\
\hline
\end{tabular}

Gene identification plays a key role in the exploration of angiogenesis and in discovering novel therapeutic targets for anti-angiogenesis drugs. Zebrafish are compliant to genetic manipulation at low cost and within a short time. In this manner, a number of signaling pathways for angiogenesis and various targets for drug treatment have been identified over the past few years. Targeted gene knockdown of TNFRSF1B in zebrafish was found to promote the apoptotic program, and knockdown of TNFRSF1A, or up-regulation of NF- $\mathrm{kB}$, prevented endothelial cell apoptosis, suggesting that TNFRSF1A and TNFRSF1B were involved in the signaling pathways of angiogenesis [81]. In another study, a silencing of LIM kinases in pancreatic cancer tissues resulted in a decrease of angiogenesis in zebrafish [82]. These data suggest new therapeutic targets for the control of the tumor-driven angiogenesis process.

Compared with other angiogenesis models such as the chorioallantoic membrane of the chicken embryo, zebrafish show their superiority with regard to modeling the in vivo environment, compliance in genetic manipulation, and allowing clear observation of the interaction between tumor cells and neonatal micrangium.

\section{Tumor metastasis in zebrafish}

The large amount of evidence from various studies has clarified that metastasis is a dynamic, complex, and multi-step process that includes tumor cells penetrating into the circulatory system, spreading to distant tissues, engrafting in the parenchyma, and developing in the graft area [83]. An insight into the mechanism of tumor metastasis is conducive to the discovery of anti-tumor drugs and the improvement of clinical treatments. Much of the previous analysis of metastasis conducted in in vitro cell systems had obvious weaknesses because the complete metastasis process cannot be abstracted away from the in vivo environment and vascular system. In vivo mouse models also have significant disadvantages: 1) it is difficult to evaluate the early stage of metastasis; 2) the complete process of metastasis in a mouse requires a long period of time; 3 ) real-time imaging of minute tumor lesions in deep tissues is impossible without termination and autopsy; 4) immunodeficiency mice may still have a residual anti-tumor competence that can prevent tumor cell metastasis [84]; 5) mice require feeding at high cost throughout the experiment. 
The cancer model of zebrafish overcomes the drawbacks of murine xenograft models and shows several exceptional strengths. The adaptive immune system in zebrafish larvae is not completely developed until 14 DPF so that most transplanted cancer cells can survive and metastasize [85]. The transparent body of zebrafish enables the clear observation of tumor metastasis under the microscope. In the transparent casper line, the dynamic and spatial characteristics of micrometastases can be real-time imaged at the single cell level [8]. In order to highlight metastasis in zebrafish, tumor cells can be stained by a chemical dyestuff (such as CM-Dil) or labeled by red fluorescent protein (RFP) [86]. By injecting red fluorescent mammalian tumor cells into the $\mathrm{Tg}$ (fli1: EGFP) transgenic zebrafish, in which vascular endothelial cells are labeled by green fluorescent protein, both the process of tumor cell metastasis and changes in the vascular system can be clearly seen throughout the body. In addition, cancer stem cells are too few in number to be transplanted in mammalian models but zebrafish are small enough for such xenografting, and the rapid progress of metastasis in zebrafish is able to be observed within 2 days after injection [65].

Zebrafish provide an experimentally tractable animal model for the identification of suppressing or promoting factors in metastasis. By transplanting RFP expressing U87 glioma stem cells (GSCs) into the yolk sac of $\operatorname{Tg}$ (fli1:EGFP) ${ }^{\mathrm{y} 1}$ zebrafish embryos, the different invasive stages of GSCs, such as approaching, clustering, invading, migrating, and transmigrating, can be clearly observed at 2 days post-injection [65]. In this experiment, invasive GSCs were found to have MMP-9 high expression in common and treatment with the MMP-9 inhibitor significantly decreased the percentage of invasive cells in the embryos [65]. In an experiment on hypoxia, DiI-labeled tumor cells were injected into the perivitelline space of $48 \mathrm{HPF}$ embryos, which were subsequently placed in hypoxic water for 3 days. A significant increase in metastasis and angiogenesis was detected using a fluorescent microscope at the single-cell level $[87,88]$. Tumor cells and immune cells have been co-implanted in the same zebrafish to investigate the interactions in the tumor microenvironment [89]. The co-implanting of DiI-labeled tumor cells and DiDlabeled tumor-associated macrophages (TAM) relates to an increase in metastasis in zebrafish, and their association could be detected in overlapping colors [89].

The signaling pathway for metastasis has been evaluated in the zebrafish model. The technology of target knockdown of proteins involved in the signaling pathway with chemical inhibition or small interfering RNA is not new in zebrafish. The TGF-beta signaling pathway was found to control human breast cancer metastasis in zebrafish. After treatment with the TGF-beta signaling pathway inhibitor, the invasion and metastasis processes in zebrafish were inhibited significantly [70, 90].

\section{Drug screening in zebrafish}

The effects of molecule antineoplastic drugs have often been detected by biochemical assays or in cell line models, but the outcomes were unsatisfactory. Because of a lack of a complete biologic context in the screening process, the identified active compounds were often ineffective when applied in a vertebrate model. At this point, a whole animal screen sheds valuable information on anti-tumor effects, organ toxicity, and pharmacokinetic data based on the entire organism [91]. However, mice are fiscally prohibitive for large-scale screen. Zebrafish, on the other hand, have emerged as a powerful platform for use in highthroughput antineoplastic drug screening on the strength of the following advantages. A pair of zebrafish produce hundreds of embryos a week, and larvae have a small size that can be arrayed in a 96-well plate, which greatly decreases the cost of maintaining them in the laboratory. Drug treatments can be easily achieved by merely adding the medicine to the aqueous environment. In addition, the transparent zebrafish body enables the real-time noninvasive imaging of anti-tumor effects and drug toxicity.

Most types of cancer can be modeled in zebrafish, thus zebrafish can be used to assess the anti-tumor effects of chemotherapeutic drugs. The growth of tumor cells and degree of invasion are the main concerned outcomes. Over the past 5 years, several large chemical screens have been performed in zebrafish. The anti-melanoma chemical genetic screen is one of the best representations. To our knowledge, the propagation of melanoma is critically related to the neural crest lineage. 2,000 chemicals were screened to identify inhibitors of the neural crest lineage in zebrafish embryos, and the selected chemicals were tested for effects in melanoma. Leflunomide, an inhibitor of dihydroorotate dehydrogenase, was found to inhibit the development of both neural crest and human melanoma. This screen shed light on the important role of zebrafish in antineoplastic drug discovery [92].

An anti-leukemia compound screen was performed in zebrafish in 2012. Zebrafish show a striking similarity in the hematopoietic system development with humans, and almost all human adult blood lineages have corresponding homologous cell lines in zebrafish. For this reason, effective hematopoietic drugs in zebrafish may serve the same function in humans. More than 25,000 small compounds were identified in this drug screen and, finally, a compound called lenaldekar (LDK) was found to be able to induce long-term remission in adult zebrafish with T-cell acute lymphoblastic leukemia (T-ALL). A subsequent study showed that LDK had a generalized antileukemia effect not only to T-ALL but also to several diverse leukemias such as B-ALL and CML [93, 94]. 
Anti-angiogenesis drugs have been screened in zebrafish. Following a screen of 288 new compounds, two kinase inhibitor compounds were found to have anti-angiogenic properties and a phosphorylase kinase subunit G1 (PhKG1) was identified as the kinase target [95]. In a similar way, rosuvastatin was identified as inhibiting the angiogenesis in developing zebrafish embryos [96]. Anti-lymphatic drug compounds were also identified in zebrafish. Four compounds previously used in humans were found to have anti-lymphatic activity in zebrafish [97]. These studies demonstrate that zebrafish provide an effective utility platform for large-scale antineoplastic drug screens and medicine efficacy detection.

Zebrafish have been used to identify compounds that work in the genetic signaling pathways of carcinogenesis. The bmyb gene is important for controlling the mitotic checkpoint and is connected with cancer susceptibility [32]. In order to identify the drug function of small molecules in the bmyb pathway, 16,000 compounds were tested. A compound named persynthamide was noted to have an inhibiting effect in bmyb-dependent mitotic defects and reduced the incidence of tumors in zebrafish [39].

Antineoplastic drug toxicity can be observed over a short period because of the rapid development of zebrafish. In a screen for detecting the inner ear hair cell toxicity of anti-tumor drugs, 13 out of 88 anti-tumor drugs, and 5 out of 10 drug combinations, were authenticated ototoxic. In addition, dose-response studies were performed on these detected drugs [98]. Several outcomes were usually detected to assess the toxic effect of antineoplastic drugs, such as cell damage, development process, and vitality. Drug toxicity screening has an important significance in selecting the appropriate therapy in clinical cancer treatments.

\section{Discussion}

Considering the past two decades, it is easy to see that enormous progress has taken place with regard to zebrafish in terms of modeling human cancers. The wide use of zebrafish sheds greater light on the investigation of gene functions, tumor angiogenesis, tumor metastasis, and the discovery of antineoplastic drugs in cancer research (Fig. 1).

Several problems often trouble cancer researchers. Traditionally, the murine system is the most utilized animal system for studying human cancers. However, the long gestation time greatly lengthens the experimental procedure. In addition, real-time imaging of minute tumor lesions in deep tissues is difficult in a murine system unless autopsy is performed. The high cost of a murine system also rejects the high-throughput screening of drug discoveries.

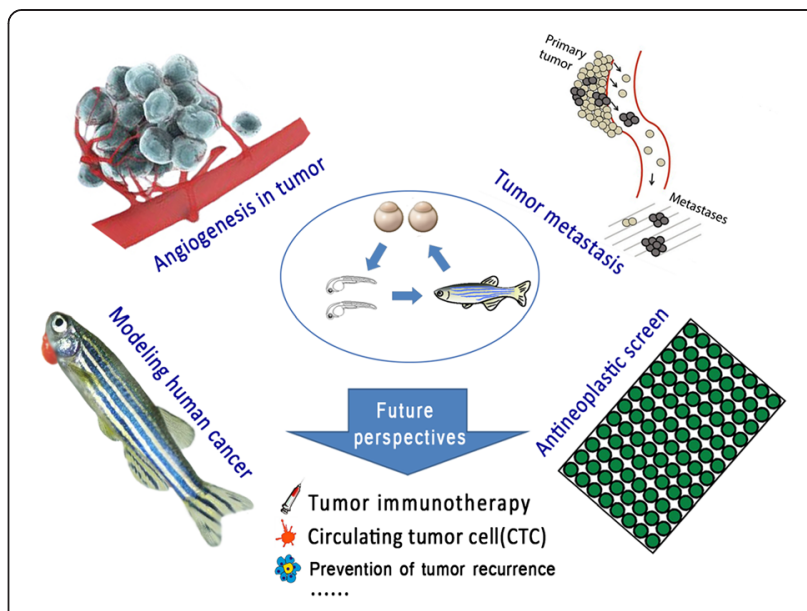

Fig. 1 The main fields of application of zebrafish in cancer research

There is no doubt that zebrafish will play an increasingly significant role in cancer research. Zebrafish have several prominent advantages in modeling human cancers, such as rapid development, a transparent body, high genetic homology, and ease of genetic manipulation. These unique advantages enable primary studies to be assumed before further verification using costly murine systems. Indeed, the ease of establishing a cancer model and the real-time observation of tumor progression in zebrafish greatly improves the efficiency of the experiment. Zebrafish provide a good model for researching the mechanisms of tumor angiogenesis and metastasis because the newly-formed vessels and metastatic tumor cells can be clearly marked and real-time observed through the transparent body. Additionally, the high reproductive rate and low financial cost of zebrafish enables high-throughput screening of anti-tumor drugs.

However, as an underdeveloped model organism, several weaknesses limit the application of zebrafish in the research community. Only a few versatile tools and validated reagents are suitable for use in zebrafish, in comparison with traditional mammalian models. This prevents us from exploring the details of the molecular and cellular mechanisms involved in cancer development. Zebrafish also have many duplicate genes that significantly complicate genetic manipulation. Forward genetic manipulation is not able to completely knock out both copies of the target genes, and any gene copies remaining will render the phenotype unchanged. CRISPR, a newly developed reverse genetic technology, shows its superiority to overcome this difficulty, as it has the property to remove multiple genes thereby reveal phenotypes [99]. In addition, it is difficult to give some water-insoluble drugs to zebrafish because the carrier solvents may be toxic before the drugs dissolve. Moreover, the embryos need to be raised in water maintained at $28{ }^{\circ} \mathrm{C}$, and this temperature may not be optimum for the metabolism of mammalian tumor cells 
(which require $37^{\circ} \mathrm{C}$ ). Therefore, raising the incubation temperature to $34{ }^{\circ} \mathrm{C}$ may offer an effective compromise. As previously reported, maintaining embryos with transplanted tumor cells in $34{ }^{\circ} \mathrm{C}$ water did not affect the vitality of the embryos or the growth of tumor cells [100].

This paper systematically expounds the significance of zebrafish within the field of cancer research. It is a comprehensive summary of the various uses of zebrafish within the field and forecasts a more extensive application of them in the future. Tumor immunotherapy represents a new prevailing therapy in the clinical treatment of tumors. Zebrafish share a similar immune system with humans, so there is, therefore, the prospect of them being used to research the curative effects of tumor immunotherapy. Several other areas, such as tumor recurrence and circulating tumor cells research, will also require a zebrafish model for further exploration of the mechanism and physiologic processes.

In conclusion, zebrafish are increasingly becoming a superior vertebrate model for cancer research and can be expected to provide further contributions to our deeper understanding of the mechanisms of genetic function, angiogenesis, metastasis, and antineoplastic drug screening in the near future.

\section{Competing interests}

The authors declare that they have no competing interests.

\section{Authors' contributions}

Ye J designed the study and reviewed the literature. Huang J participated in the design of the study and modified the manuscript. Zhao $S$ participated in the review of the literature and drafted the manuscript. All authors read and approved the final manuscript.

\section{Acknowledgements}

This work was supported by grants from the National Natural Science Foundation of China (No. 81402324), Zhejiang Provincial Natural Science Foundation of China (No. LY14H160027). This work was also funded by the Zhejiang Province Key Discipline of Traditional Chinese Medicine(2012-XKA27, JH).

Received: 22 June 2015 Accepted: 28 July 2015

Published online: 12 August 2015

\section{References}

1. Gore AV, Monzo K, Cha YR, Pan W, Weinstein BM. Vascular development in the zebrafish. Cold Spring Harb Perspect Med. 2012;2(5):a006684.

2. Kanungo J, Cuevas E, Ali SF, Paule MG. Zebrafish Model in Drug Safety Assessment. Curr Pharm Des. 2014;20(34):5416-29.

3. Kalueff AV, Stewart AM, Gerlai R. Zebrafish as an emerging model for studying complex brain disorders. Trends Pharmacol Sci. 2014;35(2):63-75.

4. Guyon JR, Steffen LS, Howell MH, Pusack TJ, Lawrence C, Kunkel LM. Modeling human muscle disease in zebrafish. Biochim Biophys Acta. 2007;1772(2):205-15.

5. Weinstein B. Vascular cell biology in vivo: a new piscine paradigm? Trends Cell Biol. 2002;12(9):439-45

6. Lieschke GJ, Oates AC, Crowhurst MO, Ward AC, Layton JE. Morphologic and functional characterization of granulocytes and macrophages in embryonic and adult zebrafish. Blood. 2001;98(10):3087-96.

7. Santoriello C, Zon LI. Hooked! Modeling human disease in zebrafish. J Clin Invest. 2012;122(7):2337-43.

8. White RM, Sessa A, Burke C, Bowman T, LeBlanc J, Ceol C, et al. Transparent adult zebrafish as a tool for in vivo transplantation analysis. Cell Stem Cell. 2008;2(2):183-9
9. Liu J, Stainier DY. Zebrafish in the study of early cardiac development. Circ Res. 2012;110(6):870-4.

10. Bakkers J. Zebrafish as a model to study cardiac development and human cardiac disease. Cardiovasc Res. 2011;91(2):279-88.

11. Spitsbergen J. Imaging neoplasia in zebrafish. Nat Methods. 2007:4(7):548-9.

12. Blackburn JS, Liu S, Raimondi AR, Ignatius MS, Salthouse CD, Langenau DM. High-throughput imaging of adult fluorescent zebrafish with an LED fluorescence macroscope. Nature Protocols. 2011;6(2):229-41.

13. Rekha RD, Amali AA, Her GM, Yeh YH, Gong HY, Hu SY, et al. Thioacetamide accelerates steatohepatitis, cirrhosis and HCC by expressing HCV core protein in transgenic zebrafish Danio rerio. Toxicology. 2008;243(1-2):11-22.

14. Park SW, Davison JM, Rhee J, Hruban RH, Maitra A, Leach SD. Oncogenic KRAS induces progenitor cell expansion and malignant transformation in zebrafish exocrine pancreas. Gastroenterology. 2008;134(7):2080-90.

15. Fleming A, Jankowski J, Goldsmith P. In vivo analysis of gut function and disease changes in a zebrafish larvae model of inflammatory bowel disease: a feasibility study. Inflamm Bowel Dis. 2010;16(7):1162-72.

16. Cinaroglu A, Gao C, Imrie D, Sadler KC. Activating transcription factor 6 plays protective and pathological roles in steatosis due to endoplasmic reticulum stress in zebrafish. Hepatology. 2011:54(2):495-508.

17. Passeri MJ, Cinaroglu A, Gao C, Sadler KC. Hepatic steatosis in response to acute alcohol exposure in zebrafish requires sterol regulatory element binding protein activation. Hepatology. 2009;49(2):443-52.

18. Ziv L, Muto A, Schoonheim PJ, Meijsing SH, Strasser D, Ingraham HA, et al. An affective disorder in zebrafish with mutation of the glucocorticoid receptor. Mol Psychiatry. 2013;18(6):681-91.

19. Stewart AM, Nguyen M, Wong K, Poudel MK, Kalueff AV. Developing zebrafish models of autism spectrum disorder (ASD). Prog Neuropsychopharmacol Biol Psychiatry. 2014;50:27-36.

20. Burgess HA, Granato M. Sensorimotor gating in larval zebrafish. J Neurosci. 2007;27(18):4984-94.

21. Parsons MJ, Campos I, Hirst EM, Stemple DL. Removal of dystroglycan causes severe muscular dystrophy in zebrafish embryos. Development. 2002;129(14):3505-12.

22. Niethammer P, Grabher C, Look AT, Mitchison TJ. A tissue-scale gradient of hydrogen peroxide mediates rapid wound detection in zebrafish. Nature. 2009;459(7249):996-9.

23. Mizgirev I, Revskoy S. Generation of clonal zebrafish lines and transplantable hepatic tumors. Nat Protoc. 2010;5(3):383-94.

24. Basten SG, Davis EE, Gillis AJ, van Rooijen E, Stoop H, Babala N, et al. Mutations in LRRC50 predispose zebrafish and humans to seminomas. PLoS Genet. 2013;9(4), e1003384.

25. Lam SH, Wu YL, Vega VB, Miller LD, Spitsbergen J, Tong Y, et al. Conservation of gene expression signatures between zebrafish and human liver tumors and tumor progression. Nat Biotechnol. 2006;24(1):73-5.

26. Mizgireuv IV, Revskoy SY. Transplantable tumor lines generated in clonal zebrafish. Cancer Res. 2006;66(6):3120-5

27. Spitsbergen JM, Tsai HW, Reddy A, Miller T, Arbogast D, Hendricks JD, et al Neoplasia in zebrafish (Danio rerio) treated with N-methyl-N'-nitro-Nnitrosoguanidine by three exposure routes at different developmental stages. Toxicol Pathol. 2000;28(5):716-25.

28. Spitsbergen JM, Tsai HW, Reddy A, Miller T, Arbogast D, Hendricks JD, et al. Neoplasia in zebrafish (Danio rerio) treated with 7,12-dimethylbenz[a]anthracene by two exposure routes at different developmental stages. Toxicol Pathol. 2000;28(5):705-15.

29. Feitsma H, Cuppen E. Zebrafish as a cancer model. Mol Cancer Res. 2008;6(5):685-94.

30. Mirbahai L, Williams TD, Zhan H, Gong Z, Chipman JK. Comprehensive profiling of zebrafish hepatic proximal promoter CpG island methylation and its modification during chemical carcinogenesis. BMC Genomics. 2011;12:3.

31. Mizgireuv IV, Majorova IG, Gorodinskaya VM, Khudoley W, Revskoy SY. Carcinogenic effect of $\mathrm{N}$-nitrosodimethylamine on diploid and triploid zebrafish (Danio rerio). Toxicol Pathol. 2004;32(5):514-8.

32. Shepard JL, Amatruda JF, Stern HM, Subramanian A, Finkelstein D, Ziai J, et al. A zebrafish bmyb mutation causes genome instability and increased cancer susceptibility. Proc Natl Acad Sci U S A. 2005;102(37):13194-9.

33. Santhakumar K, Judson EC, Elks PM, McKee S, Elworthy S, van Rooijen E, et al. A zebrafish model to study and therapeutically manipulate hypoxia signaling in tumorigenesis. Cancer Res. 2012;72(16):4017-27. 
34. Hurwitz H, Fehrenbacher L, Novotny W, Cartwright T, Hainsworth J, Heim W, et al. Bevacizumab plus irinotecan, fluorouracil, and leucovorin for metastatic colorectal cancer. N Engl J Med. 2004;350(23):2335-42.

35. Kuroyanagi M, Katayama T, Imai T, Yamamoto Y, Chisada S, Yoshiura Y, et al. New approach for fish breeding by chemical mutagenesis: establishment of TILLING method in fugu (Takifugu rubripes) with ENU mutagenesis. BMC Genomics. 2013;14:786.

36. Wienholds E, van Eeden F, Kosters M, Mudde J, Plasterk RH, Cuppen E. Efficient target-selected mutagenesis in zebrafish. Genome Res. 2003;13(12):2700-7.

37. Da Costa MM, Allen CE, Higginbottom A, Ramesh T, Shaw PJ, McDermott CJ. A new zebrafish model produced by TILLING of SOD1-related amyotrophic lateral sclerosis replicates key features of the disease and represents a tool for in vivo therapeutic screening. Dis Model Mech. 2014;7(1):73-81.

38. Stemple DL. TILLING-a high-throughput harvest for functional genomics. Nat Rev Genet. 2004;5(2):145-50.

39. Stern HM, Murphey RD, Shepard $J L$, Amatruda JF, Straub $C T$, Pfaff $K L$, et al. Small molecules that delay $S$ phase suppress a zebrafish bmyb mutant. Nat Chem Biol. 2005;1(7):366-70.

40. Huang $P$, Zhu Z, Lin S, Zhang B. Reverse genetic approaches in zebrafish. J Genet Genomics. 2012;39(9):421-33.

41. Storer NY, Zon LI. Zebrafish models of p53 functions. Cold Spring Harb Perspect Biol. 2010;2(8):a001123.

42. Phelps RA, Chidester S, Dehghanizadeh S, Phelps J, Sandoval IT, Rai K, et al. A two-step model for colon adenoma initiation and progression caused by APC loss. Cell. 2009;137(4):623-34.

43. Shin J, Padmanabhan A, de Groh ED, Lee JS, Haidar S, Dahlberg S, et al. Zebrafish neurofibromatosis type 1 genes have redundant functions in tumorigenesis and embryonic development. Dis Model Mech. 2012;5(6):881-94

44. Shive HR, West RR, Embree LJ, Golden CD, Hickstein DD. brca2 in zebrafish ovarian development, spermatogenesis, and tumorigenesis. Proc Natl Acad Sci U S A. 2010;107(45):19350-5

45. Neumann JC, Dovey JS, Chandler GL, Carbajal L, Amatruda JF. Identification of a heritable model of testicular germ cell tumor in the zebrafish. Zebrafish. 2009;6(4):319-27.

46. Reischauer S, Levesque MP, Nüsslein-Volhard C, Sonawane M. Lgl2 executes its function as a tumor suppressor by regulating ErbB signaling in the zebrafish epidermis. PLoS Genet. 2009;5(11), e1000720.

47. Shepard JL, Amatruda JF, Finkelstein D, Ziai J, Finley KR, Stern HM, et al. A mutation in separase causes genome instability and increased susceptibility to epithelial cancer. Genes Dev. 2007;21(1):55-9.

48. Yang F, Xiong J, Jia XE, Gu ZH, Shi JY, Zhao Y, et al. GSTT1 deletion is related to polycyclic aromatic hydrocarbons-induced DNA damage and lymphoma progression. PLoS One. 2014;9(2), e89302.

49. Gutierrez A, Grebliunaite R, Feng H, Kozakewich E, Zhu S, Guo F, et al. Pten mediates Myc oncogene dependence in a conditional zebrafish model of T cell acute lymphoblastic leukemia. J Exp Med. 2011;208(8):1595-603.

50. Choorapoikayil S, Kuiper RV, de Bruin A, den Hertog J. Haploinsufficiency of the genes encoding the tumor suppressor Pten predisposes zebrafish to hemangiosarcoma. Dis Model Mech. 2012;5(2):241-7.

51. Langenau DM, Traver D, Ferrando AA, Kutok JL, Aster JC, Kanki JP, et al. Myc-induced T cell leukemia in transgenic zebrafish. Science. 2003;299(5608):887-90.

52. Li Z, Zhan H, Zeng Z, Li C, Spitsbergen JM, Meierjohann S, et al. Inducible and repressable oncogene-addicted hepatocellular carcinoma in Tet-on xmrk transgenic zebrafish. J Hepatol. 2012;56(2):419-25.

53. Li Z, Zheng W, Wang Z, Zeng Z, Zhan H, Li C, et al. A transgenic zebrafish liver tumor model with inducible Myc expression reveals conserved Myc signatures with mammalian liver tumors. Dis Model Mech. 2013;6(2):414-23.

54. Nguyen AT, Emelyanov A, Koh CH, Spitsbergen JM, Parinov S, Gong Z. An inducible kras(V12) transgenic zebrafish model for liver tumorigenesis and chemical drug screening. Dis Model Mech. 2012;5(1):63-72.

55. Zheng W, Li Z, Nguyen AT, Li C, Emelyanov A, Gong Z. Xmrk, kras and myc transgenic zebrafish liver cancer models share molecular signatures with subsets of human hepatocellular carcinoma. PLoS One. 2014;9(3), e91179.

56. Zhu S, Lee JS, Guo F, Shin J, Perez-Atayde AR, Kutok JL, et al. Activated ALK collaborates with MYCN in neuroblastoma pathogenesis. Cancer Cell. 2012;21(3):362-73.
57. Storer NY, White RM, Uong A, Price E, Nielsen GP, Langenau DM, et al. Zebrafish rhabdomyosarcoma reflects the developmental stage of oncogene expression during myogenesis. Development. 2013;140(14):3040-50.

58. Chu CY, Chen CF, Rajendran RS, Shen CN, Chen TH, Yen CC, et al. Overexpression of Akt1 enhances adipogenesis and leads to lipoma formation in zebrafish. PLoS One. 2012;7(5), e36474.

59. Lu JW, Yang WY, Tsai SM, Lin YM, Chang PH, Chen JR, et al. Liver-specific expressions of $\mathrm{HBx}$ and src in the p53 mutant trigger hepatocarcinogenesis in zebrafish. PLoS One. 2013;8(10), e76951.

60. Dovey M, White RM, Zon LI. Oncogenic NRAS cooperates with p53 loss to generate melanoma in zebrafish. Zebrafish. 2009;6(4):397-404.

61. Leacock SW, Basse AN, Chandler GL, Kirk AM, Rakheja D, Amatruda JF. A zebrafish transgenic model of Ewing's sarcoma reveals conserved mediators of EWS-FLI1 tumorigenesis. Dis Model Mech. 2012;5(1):95-106.

62. Ju B, Spitsbergen J, Eden CJ, Taylor MR, Chen W. Co-activation of hedgehog and AKT pathways promote tumorigenesis in zebrafish. Mol Cancer. 2009;8:40

63. Smith AC, Raimondi AR, Salthouse CD, Ignatius MS, Blackburn JS, Mizgirev IV, et al. High-throughput cell transplantation establishes that tumorinitiating cells are abundant in zebrafish T-cell acute lymphoblastic leukemia. Blood. 2010;115(16):3296-303.

64. Lee LM, Seftor EA, Bonde G, Cornell RA, Hendrix MJ. The fate of human malignant melanoma cells transplanted into zebrafish embryos: assessment of migration and cell division in the absence of tumor formation. Dev Dyn. 2005;233(4):1560-70.

65. Yang XJ et al. A novel zebrafish xenotransplantation model for study of glioma stem cell invasion. PLoS One. 2013;8(4), e61801.

66. Hou Y, Chu M, Du FF, Lei JY, Chen Y, Zhu RY, et al. Recombinant disintegrin domain of ADAM15 inhibits the proliferation and migration of Bel-7402 cells. Biochem Biophys Res Commun. 2013;435(4):640-5.

67. Moshal KS, Ferri-Lagneau KF, Haider J, Pardhanani P, Leung T. Discriminating different cancer cells using a zebrafish in vivo assay. Cancers (Basel). 2011;3(4):4102-13.

68. Weiss FU, Marques IJ, Woltering JM, Vlecken DH, Aghdassi A, Partecke LI, et al. Retinoic acid receptor antagonists inhibit miR-10a expression and block metastatic behavior of pancreatic cancer. Gastroenterology. 2009;137(6):2136-45.

69. Latifi A, Abubaker K, Castrechini N, Ward AC, Liongue C, Dobill F, et al. Cisplatin treatment of primary and metastatic epithelial ovarian carcinomas generates residual cells with mesenchymal stem cell-like profile. J Cell Biochem. 2011;112(10):2850-64.

70. Drabsch Y, He S, Zhang L, Snaar-Jagalska BE, ten Dijke P. Transforming growth factor-beta signalling controls human breast cancer metastasis in a zebrafish xenograft model. Breast Cancer Res. 2013;15(6):R106.

71. Wagner DS, Delk NA, Lukianova-Hleb EY, Hafner JH, Farach-Carson MC, Lapotko DO. The in vivo performance of plasmonic nanobubbles as cell theranostic agents in zebrafish hosting prostate cancer xenografts. Biomaterials. 2010;31(29):7567-74.

72. Jo DH, Son D, Na Y, Jang M, Choi JH, Kim JH, et al. Orthotopic transplantation of retinoblastoma cells into vitreous cavity of zebrafish for screening of anticancer drugs. Mol Cancer. 2013;12:71.

73. Zhang B, Shimada Y, Kuroyanagi J, Umemoto N, Nishimura Y, Tanaka T. Quantitative phenotyping-based in vivo chemical screening in a zebrafish model of leukemia stem cell xenotransplantation. PLoS One. 2014;9(1), e85439.

74. Bellou S, Pentheroudakis G, Murphy C, Fotsis T. Anti-angiogenesis in cancer therapy: Hercules and hydra. Cancer Lett. 2013;338(2):219-28.

75. Yue GG, Fan JT, Lee JK, Zeng GZ, Ho TW, Fung KP, et al. Cyclopeptide RA-V inhibits angiogenesis by down-regulating ERK1/2 phosphorylation in HUVEC and HMEC-1 endothelial cells. Br J Pharmacol. 2011;164(7):1883-98.

76. Hasan J, Shnyder SD, Bibby M, Double JA, Bicknel R, Jayson GC. Quantitative angiogenesis assays in vivo-a review. Angiogenesis. 2004;7(1):1-16.

77. Tobia C, De Sena G, Presta M. Zebrafish embryo, a tool to study tumor angiogenesis. Int J Dev Biol. 2011;55(4-5):505-9.

78. Goldsmith JR, Jobin C. Think small: zebrafish as a model system of human pathology. J Biomed Biotechnol. 2012;2012:817341.

79. Kamei M, Isogai S, Pan W, Weinstein BM. Imaging blood vessels in the zebrafish. Methods Cell Biol. 2010;100:27-54.

80. Nicoli S, Ribatti D, Cotelli F, Presta M. Mammalian tumor xenografts induce neovascularization in zebrafish embryos. Cancer Res. 2007;67(7):2927-31.

81. Espin R, Roca FJ, Candel S, Sepulcre MP, González-Rosa JM, Alcaraz-Pérez F, et al. TNF receptors regulate vascular homeostasis in zebrafish through a caspase-8, caspase-2 and P53 apoptotic program that bypasses caspase-3. Dis Model Mech. 2013;6(2):383-96. 
82. Vlecken DH, Bagowski CP. LIMK1 and LIMK2 are important for metastatic behavior and tumor cell-induced angiogenesis of pancreatic cancer cells. Zebrafish. 2009;6(4):433-9.

83. Eccles SA, Welch DR. Metastasis: recent discoveries and novel treatment strategies. Lancet. 2007:369(9574):1742-57.

84. Teng Y, Xie X, Walker S, White DT, Mumm JS, Cowell JK. Evaluating human cancer cell metastasis in zebrafish. BMC Cancer. 2013;13:453.

85. Traver D, Herbomel P, Patton EE, Murphey RD, Yoder JA, Litman GW, et al. The zebrafish as a model organism to study development of the immune system. Adv Immunol. 2003;81:253-330.

86. Marques IJ, Weiss FU, Vlecken DH, Nitsche C, Bakkers J, Lagendijk AK, et al. Metastatic behaviour of primary human tumours in a zebrafish xenotransplantation model. BMC Cancer. 2009;9:128.

87. Kabbinavar F, Hurwitz HI, Fehrenbacher L, Meropol NJ, Novotny WF, Lieberman $\mathrm{G}$, et al. Phase II, randomized trial comparing bevacizumab plus fluorouracil (FU)/leucovorin (LV) with FU/LV alone in patients with metastatic colorectal cancer. J Clin Oncol. 2003;21(1):60-5.

88. Lee SL, Rouhi P, Dahl Jensen L, Zhang D, Ji H, Hauptmann G, et al. Hypoxia-induced pathological angiogenesis mediates tumor cell dissemination, invasion, and metastasis in a zebrafish tumor model. Proc Natl Acad Sci U S A. 2009;106(46):19485-90.

89. Wang J, Cao Z, Zhang XM, Nakamura M, Sun M, Hartman J, et al. Novel mechanism of macrophage-mediated metastasis revealed in a zebrafish model of tumor development. Cancer Res. 2015;75(2):306-15.

90. Li Y, Drabsch Y, Pujuguet P, Ren J, van Laar T, Zhang L, et al. Genetic depletion and pharmacological targeting of alphav integrin in breast cancer cells impairs metastasis in zebrafish and mouse xenograft models. Breast Cancer Res. 2015:17(1):28.

91. Ghotra VP, He S, de Bont H, van der Ent W, Spaink HP, van de Water B, et al. Automated whole animal bio-imaging assay for human cancer dissemination. PLoS One. 2012;7(2), e31281.

92. White RM, Cech J, Ratanasirintrawoot S, Lin CY, Rahl PB, Burke CJ, et al. $\mathrm{DHODH}$ modulates transcriptional elongation in the neural crest and melanoma. Nature. 2011;471(7339):518-22.

93. Clements WK, Traver D. Fish pharming: zebrafish antileukemia screening. Blood. 2012;119(24):5614-5.

94. Ridges $\mathrm{S}$, Heaton $\mathrm{WL}$, Joshi $\mathrm{D}$, Choi $\mathrm{H}$, Eiring $\mathrm{A}$, Batchelor $\mathrm{L}$, et al. Zebrafish screen identifies novel compound with selective toxicity against leukemia. Blood. 2012:119(24):5621-31.

95. Camus S, Quevedo C, Menéndez S, Paramonov I, Stouten PF, Janssen RA. Identification of phosphorylase kinase as a novel therapeutic target through high-throughput screening for anti-angiogenesis compounds in zebrafish. Oncogene. 2012;31(39):4333-42.

96. Wang C, Tao W, Wang Y, Bikow J, Lu B, Keating A, et al. Rosuvastatin, identified from a zebrafish chemical genetic screen for antiangiogenic compounds, suppresses the growth of prostate cancer. Eur Urol. 2010;58(3):418-26.

97. Astin JW, Jamieson SM, Eng TC, Flores MV, Misa JP, Chien A, et al. An in vivo antilymphatic screen in zebrafish identifies novel inhibitors of mammalian lymphangiogenesis and lymphatic-mediated metastasis. Mol Cancer Ther. 2014; 13(10):2450-62

98. Hirose $Y$, Simon JA, Ou HC. Hair cell toxicity in anti-cancer drugs: evaluating an anti-cancer drug library for independent and synergistic toxic effects on hair cells using the zebrafish lateral line. J Assoc Res Otolaryngol. 2011;12(6):719-28

99. Shah AN, Davey CF, Whitebirch AC, Miller AC, Moens CB. Rapid reverse genetic screening using CRISPR in zebrafish. Nat Methods. 2015;12(6):535-40.

100. He S, Lamers GE, Beenakker JW, Cui C, Ghotra VP, Danen EH, et al. Neutrophil-mediated experimental metastasis is enhanced by VEGFR inhibition in a zebrafish xenograft model. J Pathol. 2012;227(4):431-45.

\section{Submit your next manuscript to BioMed Central and take full advantage of:}

- Convenient online submission

- Thorough peer review

- No space constraints or color figure charges

- Immediate publication on acceptance

- Inclusion in PubMed, CAS, Scopus and Google Scholar

- Research which is freely available for redistribution

Submit your manuscript at www.biomedcentral.com/submit 